\title{
Pediatric out-of-hospital deaths following hospital discharge: a mixed-methods study.
}

Lacey English ${ }^{1}$, Elias Kumbakumba ${ }^{2}$, Charles P Larson ${ }^{3}$, Jerome Kabakyenga ${ }^{4}$, Joel Singer ${ }^{5,6}$, Niranjan Kissoon ${ }^{3,7}$, J Mark Ansermino ${ }^{3,8}$, Hubert Wong ${ }^{5}$, Julius Kiwanuka ${ }^{2}$, MO Wiens ${ }^{5}$

1. School of Medicine, University of North Carolina at Chapel Hill, Chapel Hill, USA

2. Department of Pediatrics, Mbarara University of Science and Technology, Mbarara, Uganda

3. Center for International Child Health, BC Children's Hospital, Child and Family Research Institute, Vancouver Canada

4. Maternal, Newborn and Child Health Institute, Mbarara University of Science and Technology, Mbarara, Uganda

5. School of Population and Public Health, University of British Columbia, Vancouver, Canada

6. Canadian HIV Trials Network, St. Paul's Hospital and University of British Columbia, Vancouver, Canada

7. Department of Pediatrics, BC Children's Hospital and University of British Columbia, Vancouver, Canada

8. Department of Pediatric Anesthesiology, BC Children's Hospital and University of British Columbia, Vancouver, Canada

\begin{abstract}
Background: Out-of-hospital death among children living in resource poor settings occurs frequently. Little is known about the location and circumstances of child death following a hospital discharge.

Objectives: This study aimed to understand the context surrounding out-of-hospital deaths and the barriers to accessing timely care for Ugandan children recently discharged from the hospital.

Methods: This was a mixed-methods sub-study within a larger cohort study of post-discharge mortality conducted in the Southwestern region of Uganda. Children admitted with an infectious illness were eligible for enrollment in the cohort study, and then followed for six months after discharge. Caregivers of children who died outside of the hospital during the six month post-discharge period were eligible to participate in this sub-study. Qualitative interviews and univariate logistic regression were conducted to determine predictors of out-of-hospital deaths.

Results: Of 1,242 children discharged, 61 died during the six month post-discharge period, with most ( $\mathrm{n}=40,66 \%$ ) dying outside of a hospital. Incremental increases in maternal education were associated with lower odds of out-of-hospital death compared to hospital death (OR: 0.38, 95\% CI: 0.19 - 0.81). The qualitative analysis identified health seeking behaviors and common barriers within the post-discharge period which delayed care seeking prior to death. For recently discharged children, caregivers often expressed hesitancy to seek care following a recent episode of hospitalization.

Conclusion: Mortality following discharge often occurs outside of a hospital context. In addition to resource limitations, the health knowledge and perceptions of caregivers can be influential to timely access to care. Interventions to decrease child mortality must consider barriers to health seeking among children following hospital discharge.
\end{abstract}

Keywords: Pediatrics, post-discharge mortality, Uganda, qualitative interviews, infectious diseas.

DOI: http://dx.doi.org/10.4314/ahs.v16i4.2

Cite as: English L, Kumbakumba E, Larson CP, Kabakyenga J, Singer J, Kissoon N, Ansermino JM, Wong H, Kiwanuka J, Wiens MO. Pediatric out-of-hospital deaths following hospital discharge: a mixed-methods study. Afri Health Sci 2016;16(4): 883-891. bttp:/ / dx.doi.org/10.4314/ abs.v16i4.2

Introduction

Of the 6.3 million deaths among children under 5 years

\section{Corresponding author:}

Matthew O Wiens,

School of Population and Public Health

University of British Columbia

Vancouver, BC, Canada

Email: mowiens@interchange.ubc.ca,

mowiens@outlook.com old, over half occur due to infectious diseases. ${ }^{1}$ Some studies suggest that child mortality due to infectious diseases occurs disproportionately outside of the hospital, rather than in the hospital. A systematic review of pediatric post-discharge mortality found that rates of death after hospitalization often exceeded in-hospital mortality. Post-discharge deaths generally occurred early (within several weeks of discharge) and in many cases did not occur in hospitals. ${ }^{4} \mathrm{~A}$ recent systematic analysis of severe acute respiratory infections (ARIs) in children under 
5 years found that although most episodes $(62 \%)$ were treated in the hospital, $81 \%$ of the deaths from severe respiratory infection occurred outside of the hospital. ${ }^{2}$ This study did not identify the proportion of out of hospital deaths which at some point received hospital care. This relationship is of critical importance since previous research has shown that most children who die following discharge do not successfully return to the hospital for care prior to death. ${ }^{3}$

Post-discharge death in children is increasingly being recognized as a major contributor to overall child mortality. Several factors, including inadequate health seeking behavior and poor access to hospital care, were responsible for the high occurrence of out of hospital death among children with ARIs. ${ }^{2}$ Barriers to seeking care in Sub-Saharan Africa are complex and can include financial constraints, distance to health facilities, cultural practices, gender dynamics, limited knowledge or information, and health facility disincentives. ${ }^{5,6}$ These barriers are thought to contribute to increased child mortality, for instance long distance from a health facility increased mortality risk by $17 \%$ in Tanzania. ${ }^{7}$

Malnutrition, previous hospitalizations and HIV are often identified as key risk factors for post-discharge mortality. While these risk factors may assist clinicians in the identification of vulnerable children, many other contextual and social factors may contribute to post-discharge mortality.

Currently, little is known about the health seeking decisions made by caregivers following hospitalizations for acute illness. Further, the context surrounding out- of hospital death has not been investigated in any research of post-discharge mortality. This oversight has important implications because understanding of the circumstances of post-discharge mortality and the barriers faced by caregivers is critical in the development of interventions aimed at reducing this burden. A focus on sub-Saharan Africa will become increasingly important, as the region is projected to account for $60 \%$ of all child deaths by $2030 .{ }^{1}$ The objective of this study is to evaluate the context surrounding out of hospital deaths in children recently admitted for an infectious illness in Uganda, and to determine the most important barriers to seeking timely care in children who died out of hospital.

African Health Sciences Vol 16 Issue 4, December, 2016

\section{Methods \\ Population}

This study was conducted on a sub-set of participants of a larger cohort study focused on determining predictors of post-discharge mortality among children admitted with infectious illness. A detailed review of the methods has been previously described. ${ }^{8}$ Briefly, all children aged 6 months to 5 years admitted to Mbarara Regional Referral Hospital (MRRH) and Holy Innocents Children's Hospital $(\mathrm{HICH})$ with a suspected or confirmed infectious illness were eligible for inclusion into the main study. All enrolled children had baseline characteristics measured which included clinical, laboratory and social variables. Patients received routine care during admission and were subsequently followed-up at 6 months post-discharge for a brief questionnaire to determine health status and health seeking during the post-discharge period.

An interim analysis of this study suggested that most post-discharge deaths occurred outside of the hospital context. The study protocol was, therefore, amended to conduct detailed qualitative interviews with families of study subjects who died during this period. The purpose of these interviews was to gain further insights into the circumstances behind these out- of- hospital deaths and to determine the most important barriers to seeking hospital care during the post-discharge period from the caregiver's perspective.

\section{Eligibility}

All subjects who were enrolled in the primary study and who died outside of the hospital during the 6 month post-discharge period were eligible for re-enrollment and re-consent. This amendment was approved by the institutional review boards at the Mbarara University of Science and Technology in Mbarara, Uganda and the University of British Columbia in Vancouver, BC.

\section{Study procedure}

The families of eligible subjects who died outside of the hospital during follow-up were contacted by phone and invited to participate in this study. If verbal consent was obtained, the primary caregiver was visited at their home and formally re-consented. A research nurse trained in qualitative methods administered a structured questionnaire recorded using a digital audio recorder (Table 1). The questionnaire consisted of eight open-ended questions related to events leading up to the child's death and subsequent probes. Questions sought to explore health seeking behavior and barriers to care just prior to death. 


\section{Analysis}

Interviews were translated and transcribed from Runyankole-Rukiga into English. Thematic codebook development followed a standardized framework. ' Through an iterative process, interviews were coded and analyzed for descriptive and interpretive themes. Descriptive themes included barriers to care and health seeking behavior during the post-discharge period, while interpretive themes focused on caregiver perspectives of child death and the role of the health care system. Thematic frequencies were generated to quantify medical symptoms, health seeking behavior, and barriers to care. Common themes were summarized to describe conceptual frameworks elicited by caregivers.
To determine if the demographic, social or environmental factors reported at admission were associated with out of hospital death (vs. in-hospital death), univariate logistic regression analysis was conducted. Analyses were limited to univariate analysis due to the relatively few outcomes available. All quantitative analyses were conducted using SAS 9.3 (Carey, NC).

\section{Results}

\section{Participant characteristics}

From the 1,307 children who were enrolled in the primary cohort study, 65 died during their initial admission. Of the 61 who died within six months following discharge from the hospital, the majority $(n=40)$ died outside of the hospital without being readmitted. Among children who died at home the majority had discharge diagnoses of malaria, pneumonia, HIV and/or anemia.

\section{Table 1. Structured interview questionnaire and probes}

1. Tell me about the events leading up to the death of your child? Give as many details as you can think of.

○ What happened?

- What were the initial symptoms and how did the illness progress?

2. Did you think of seeking care for your child?

3. What were the barriers/difficulties/challenges that prevented you (the parents/guardians) from going to the hospital/health facility to seek care?

4. Where exactly did the child die?

- For instance, on the way to or at the hospital/health centre, at home, local clinic, another person's home (aunt, grandmother etc.) or with traditional healer.

5. Where was the child staying just prior to death?

○ho was looking after the child at the time of becoming sick?

6. Did you (or the person caring for the child) notice that your child was very sick on this occasion?

○ If no, did your child appear healthy/well or like usual?

- If yes, what were the things you noticed as being the signs your child was very sick?

7. What were the barriers/difficulties/challenges that prevented you (the parents/guardians) from going to the hospital/health facility to seek care?

8. Could anything have been done to help you or your child?

o If yes, in your opinion, what could have been done? 
For mothers, the mean age was 29 years, and $35(87 \%)$ had either no formal education or only primary education (Table 2).

Table 2. Participant characteristics of children who died following discharge

\begin{tabular}{|c|c|}
\hline Variable & Mean \pm SD or $N(\%)$ \\
\hline Age (months) & $19.2 \pm 14.2$ \\
\hline \multicolumn{2}{|l|}{ Sex } \\
\hline Male & $22(55.0 \%)$ \\
\hline Female & $18(45.0 \%)$ \\
\hline \multicolumn{2}{|l|}{ Discharge Diagnoses* } \\
\hline Malaria & $14(35.0 \%)$ \\
\hline Pneumonia & $16(40.0 \%)$ \\
\hline Diarrhea & $3(7.5 \%)$ \\
\hline Meningitis & $3(7.5 \%)$ \\
\hline Malnutrition & $6(15 \%)$ \\
\hline Tuberculosis & $1(2.5 \%)$ \\
\hline Respiratory infection & $2(5.0 \%)$ \\
\hline Sepsis & $2(5.0 \%)$ \\
\hline Bronchiolitis & $1(2.5 \%)$ \\
\hline Anemia & $7(17.5 \%)$ \\
\hline Other & $6(15 \%)$ \\
\hline \multicolumn{2}{|l|}{ Distance to hospital } \\
\hline$<30$ minutes & $6(15.0 \%)$ \\
\hline 30 minutes -1 hour & $7(17.5 \%)$ \\
\hline$>1$ hour & $27(67.5 \%)$ \\
\hline \multicolumn{2}{|l|}{ HIV status } \\
\hline Child HIV+ & $7(17.5 \%)$ \\
\hline Maternal HIV+ & $6(15.0 \%)$ \\
\hline Maternal age (years) & $29.0 \pm 7.25$ \\
\hline \multicolumn{2}{|l|}{ Maternal education } \\
\hline$<$ Primary 3 & $12(30.0 \%)$ \\
\hline Primary $3-$ primary 7 & $22(55.0 \%)$ \\
\hline Secondary & $5(12.5 \%)$ \\
\hline Post-secondary & $1(2.5 \%)$ \\
\hline \multicolumn{2}{|l|}{ Water supply } \\
\hline Municipal water & $6(15.0 \%)$ \\
\hline Protected spring & $4(10.0 \%)$ \\
\hline Bore hole & $6(15.0 \%)$ \\
\hline Fast running stream & $1(2.5 \%)$ \\
\hline Slow stream & $1(2.5 \%)$ \\
\hline Shallow well & $21(52.5 \%)$ \\
\hline \multicolumn{2}{|l|}{ Bed net use (child) } \\
\hline Always & $15(37.5 \%)$ \\
\hline Sometimes & $12(30.0 \%)$ \\
\hline Never & $13(32.5 \%)$ \\
\hline
\end{tabular}

The participant population was also characterized by their home environment. Most caregivers $(n=27,67.5 \%$ ) lived more than one hour from the hospital. For most, their water supply came from shallow wells, and approximately one-third of children always slept under a bed net.

\section{Post-discharge mortality}

Among 40 children who died out- of- hospital post discharge, $31(77.5 \%)$ were staying with both parents just pri-

African Health Sciences Vol 16 Issue 4, December, 2016 or to death. Over half of post-discharge child mortality occurred at home $(n=24,60 \%)$, while another one-third $(n=11,27.5 \%)$ on the way to or upon reaching a medical facility. The remaining children died at or on the way to church, to stay with family, or home from the hospital.

\section{Factors associated with out of hospital death}

Several social and environmental factors described the context within which children died outside the hospital, rather than reaching the hospital (Table 3). 
Table 3. Predictors of out of hospital death $(n=40)$ among children who died post-discharge $(n=61)$

\begin{tabular}{|c|c|c|}
\hline Variable & OR $(95 \%$ CI $)$ & p-value \\
\hline Sex $($ ref $=$ male $)$ & $1.10(0.38-3.19)$ & 0.86 \\
\hline Age (per 1 month increase) & $1.04(0.99-1.10)$ & 0.12 \\
\hline HIV positive & $1.33(0.31-5.86)$ & 0.70 \\
\hline \multicolumn{3}{|l|}{ Bed net use (ref = always) } \\
\hline Sometimes & $6.40(1.22-33.47)$ & 0.03 \\
\hline Never & $8.00(1.56-41.03)$ & 0.01 \\
\hline \multicolumn{3}{|l|}{ Maternal education $($ ref $=<\mathbf{P 3})$} \\
\hline P4 - P7 & $0.35(0.07-1.87)$ & 0.22 \\
\hline $\mathrm{S} 1$ - S6 & $0.19(0.03-1.40)$ & 0.10 \\
\hline Post-secondary & $0.04(0.00-0.54)$ & 0.02 \\
\hline Maternal education (per category increase) & $0.38(0.19-0.79)$ & 0.009 \\
\hline \multicolumn{3}{|l|}{ Distance (ref $=<\mathbf{3 0}$ minutes) } \\
\hline $30-60$ minutes & $4.00(0.62-25.96)$ & 0.15 \\
\hline$>60$ minutes & $3.20(0.92-11.12)$ & 0.07 \\
\hline \multicolumn{3}{|l|}{ Maternal HIV status (ref = negative) } \\
\hline Positive & $1.38(0.32-6.05)$ & 0.67 \\
\hline Unknown & $1.21(0.27-5.42)$ & 0.80 \\
\hline \multicolumn{3}{|l|}{ Water source ( $r e f=$ municipal water) } \\
\hline Protected spring & $12.00(1.20-120.08)$ & 0.03 \\
\hline Bore hole & $8.00(0.75-85.73)$ & 0.09 \\
\hline Fast running stream & NA & NA \\
\hline Slow stream & NA & NA \\
\hline Shallow well & $10.50(2.58-42.68)$ & 0.001 \\
\hline Early death $($ ref=<30days) & $1.92(0.65-5.68)$ & 0.24 \\
\hline Discharged against medical advice & $1.05(0.33-3.33)$ & 0.95 \\
\hline \multicolumn{3}{|l|}{ Length of stay (ref $=<3$ days) } \\
\hline 3-5 days & $1.15(0.24-5.39)$ & 0.86 \\
\hline$>5$ days & $0.72(0.20-2.59)$ & 0.61 \\
\hline
\end{tabular}

Maternal education was significantly associated with odds of out- of- hospital death, such that those with post-secondary school education had a $96 \%$ lower odds of their children dying outside the hospital than those with only some or no primary school education (OR 0.04; 95\% CI $0.00-0.59)$. The odds of the child dying outside the hospital decreased by $62 \%$ with each additional level of maternal education, from primary to secondary to post-secondary (OR 0.38; 95\% CI 0.19 - 0.81). Of borderline statistical significance, children who lived more than 1 hour from the admitting facility had an odds ratio of dying of 3.20 (95\% CI $0.92-11.12)$.

Within the home environment, bed net use and water source were also associated with increased odds of death outside the hospital. Children who never used bed nets had much higher odds of out of hospital death than those who used bed nets (OR 8.00; 95\% CI 1.56 - 41.03). Similarly, the odds of dying outside the hospital for children with a shallow well as their primary water source were 10.5 times the odds of those with municipal water as their primary source (OR 10.50; 95\% CI 2.58 - 42.68). The sex, age, and HIV status of the child did not appear to be associated with out of hospital death, although the small sample does not rule out type II error.

Among children who died, the median duration of hospital stay during the initial admission was 4.5 days, with half of all deaths occurring within 30 days following discharge. Approximately one-third of caregivers $(n=12$, $30 \%$ ) removed their child from the hospital against med- 
ical advice. However, leaving against medical advice was not associated with an increased risk of out of hospital death (OR 0.97; 95\% CI 0.30-3.11).

\section{Health seeking behavior}

Almost all caregivers considered seeking care for their child prior to death ( $\mathrm{n}=36,90 \%)$, but a small minority did not consider additional care due to monetary expense, recent discharge from the hospital, or lack of previous improvement. One caregiver explained: "Since I had just been discharged from a big hospital, [I] did not think of seeking any other care." Of the 36 caregivers who considered seeking care, only 30 caregivers actually did so. Common limitations included transportation costs and arrangements. Among caregivers who did not access care, the majority had children who died within 24 hours of noticeable symptoms. One caregiver reported, "If the sickness was not sudden, they would have taken the child back to Mbarara... but it wasn't possible because the child died within a short time."

Most caregivers sought multiple means of addressing the child's illness, including home remedies, traditional medicine, health centers, hospitals, and divine intervention.
Initial point of care, indicating the first care seeking behavior after noticing symptoms, varied among caregivers. Some sought care at hospitals, while others went to health centers or utilized traditional medicine. When people took their child to traditional healers or herbalists but noticed no improvement, they often turned to hospitals or health centers for healing. For example, one caregiver said: "Child was taken to the herbalist who excised the enlarged lymph nodes and gave herbs for the enlarged spleen. I noted no improvement, [so] decided to take him to the nearby health center."

\section{Common barriers to care}

Financial constraints were the most common barrier to seeking care at a medical facility. This barrier often presented as limited transportation options, which became particularly important when the child's health deteriorated during the night. Lack of paternal support also played a significant role in financial instability for some caregivers. Notably, if a caregiver did not observe their child improving after one or multiple visits to a medical facility, they were less likely to return just prior to the death of their child. Approximately one-third of caregivers ( $\mathrm{n}=15$, $37.5 \%$ ) reported no barriers to seeking care at a medical facility (Table 4).

Table 4. Illustrative quotes of barriers to care identified through interviews

\begin{tabular}{ll}
\hline Barriers to Care & Quote \\
\hline Monetary constraints & $\begin{array}{l}\text { I took her to the nearby clinic and was seen by a nurse who referred us to the } \\
\text { hospital. However, due to lack of money, I went back home. }\end{array}$ \\
\hline Transportation & $\begin{array}{l}\text { The cars in this place do not travel at night, starting from midnight to } 5 \text { am. Before } \\
\text { that time, the only option is hiring which was too expensive for us, so this caused a } \\
\text { delay to reach the hospital. }\end{array}$ \\
\hline Recent hospital visit & $\begin{array}{l}\text { I only delayed to take him to the hospital because I thought that maybe the sickness } \\
\text { was the effect of drugs he had received in the hospital... but he kept getting worse. }\end{array}$ \\
\hline No notable improvement & $\begin{array}{l}\text { As for the health center which was near me, I had been going there with no } \\
\text { improvement, so I did not see any need to go back. }\end{array}$ \\
\hline
\end{tabular}

Though many barriers were mentioned, two responses were commonly given when asked if anything could have been done to help the child. Caregivers focused on the need for affordable transport options and access to improved health care services. One father said "if they had fast means to take their child to hospital, maybe the child's life would have been saved." Distance and road infrastructure contributed to limited transport, as one mother described, "the road was so flooded that she failed to get means to reach the hospital in time." In addition, caregivers often felt access to better health care - through more services or larger medical centers - would have helped their child, wishing they had "the money to take [their child] to bigger hospitals for more tests."

African Health Sciences Vol 16 Issue 4, December, 2016

\section{Role of the hospital experience}

The in-patient experience reportedly had some influence on post-discharge mortality and health seeking behavior. Several caregivers reported that their child was initially discharged from the hospital while still sick and exhibiting symptoms, such as vomiting, diarrhea, severe weakness or convulsions. Among those who returned to a health facility prior to death, some felt the hospital failed to effectively care for their child, while others indicated the health care workers tried their best. Interestingly, negative in-patient experiences did not always decrease overall perceptions of the health care provided. For one family, they described how the "process of referral was delayed 
by health workers... [and] during this time the child's condition continued to worsen (convulsing and started bleeding through the nose and mouth)." However, they felt that "the health workers in the hospital did their best and gave all possible care."

Some caregivers said recent care/discharge or an impending follow-up appointment delayed them from returning, even when their child's symptoms worsened. For example, one caregiver explained: "the child developed difficulty in breathing, sharp pains and was complaining of thirst... [but] they thought the child would become better since she was on medication they were given at discharge from the hospital." Though symptoms were present, they were discounted or considered likely to improve or not recognized as severe enough to seek immediate care.

\section{Acceptance of child mortality}

Many caregivers expressed acceptance of their child's death. Half of all caregivers $(n=18,45 \%)$ said nothing could have been done to help their child. These caregivers commonly mentioned that the illness was "incurable". Solace seemed to come from the belief that all avenues of care had been pursued. Caregivers noted that "all the possible measures and assistance were given by both the health workers and the neighbors." Many tended to focus on what had been done for the child, rather than what could have been done, especially if additional measures were unavailable. Through various expressions, caregivers stated that " [their child] died a natural death like any other person can die."

Notably, religion seemed to play a dichotomous role as both curative prior to death and consoling following death. Some caregivers took their child to church or religious leaders for prayers, as a means of addressing their illness. One caregiver felt that, "if the child had been taken to a prayer palace in Kampala, he would have survived." Others used a religious framework to explain their child's passing. Many noted that "it was God's Plan that [their child] pass on at that time." To cultivate acceptance, caregivers often focused on the actions taken to help the child and acknowledged "the rest was God's Plan."

\section{Discussion}

This study examined the context of post-discharge deaths occurring outside of a hospital and determined important barriers to seeking timely medical care in critically ill children during the post-discharge period. We found that most caregivers pursued multiple avenues of care for their child, but in many cases, the child's health deteriorated quickly. Common barriers, such as limited transportation and monetary constraints, delayed care seeking prior to death. For recently discharged children, the caregivers often expressed hesitancy to seek immediate care. Upon leaving the hospital, many perceived that the child was cured or on trajectory for recovery, even with persisting symptoms. Recognition of preliminary symptoms or improved modalities of reaching care could enable earlier heath seeking behavior.

These interviews also demonstrated that health care access extends beyond resource limitations; this study identified important social and environmental risk factors associated with out- of- hospital deaths during the post-discharge period. We found that factors, such as maternal education, water source, and bed net use, were predictive of out- of- hospital death. Other home characteristics have been shown to increase the odds of under-five mortality as well, including large family size, poor birth spacing, poor sanitation, and living in a rural environment. ${ }^{10-12}$ Though these aspects may not directly influence child mortality, they likely reflect the socioeconomic status and difficult circumstances within which these families are embedded.

In addition, caregiver attitudes and perceptions were important to health seeking, as they played a significant role in conceptualizing likelihood of recovery and availability of opportunities. When asked what could have saved their child, many caregivers chose to focus on steps that were taken, resources that were accessed, or help that was given, rather than hypothetical areas of improvement. Some wished for access to better health services existing elsewhere, but few focused on potential changes in their immediate environment or health system. With a fatalistic undertone, caregivers often expressed an acceptance of death and the medical resources surrounding them. Perception of the possible or the changeable may have been stifled by previous experiences, limited control, and the larger social context. Given complex weaknesses in the health system, caregivers may not have been aware of systemic solutions to prevent these out of hospital deaths, and additional studies are needed in this area.

Studies suggest that limited access to health care in developing countries, resulting from delayed health care seeking or none at all, may be an important determinant of child mortality. ${ }^{13}$ Delayed health seeking behavior, ob- 
served in our study among others, often stems from poor symptom recognition and caregiver perceptions of health resources. ${ }^{13-16}$ Previous studies of acute child illnesses indicate that danger symptoms and disease severity are often not recognized by caregivers. ${ }^{17-19}$ A cross-sectional study in Burkina Faso found that clinically defined diarrhea was recognized by only $55 \%$ of caregivers of whom only half then sought care for their child, indicating deficits in the perceived severity of the illness. ${ }^{20}$ In addition to perceptions about the illness itself, the perceived etiology, anticipated quality of care, and estimated distance to the health facility influence health seeking behavior, but their relative contributions are less studied. ${ }^{21}$

Caregiver knowledge about health has been shown to protect against child death. ${ }^{12,22}$ Our study, among others, found that maternal education was incrementally correlated with decreased odds of out- of- hospital death and infection. ${ }^{23}$ Aligning with evidence from the literature, post-discharge interventions should be developed and implemented for children at risk of out- of- hospital death. An emphasis on developing health knowledge and perceptions of caregivers has the potential to greatly impact child disease and mortality. Given the identified barriers, delayed health seeking could be improved through providing health education to caregivers on early warning symptoms or through establishment of routine post-discharge care, utilizing the available out-patient heath infrastructure (such as post-discharge follow-up at nearby health centers).

\section{Limitations}

Some limitations to the study design should be considered when interpreting results. The sample size was relatively small for quantitative analysis, making the results vulnerable to Type II error. Therefore, only very strong associations were detected. Despite the small sample size, we found several variables with high odds ratios for outof- hospital death. Another limitation is the external validity of this study. Our sample was derived from a single region of Uganda and included only discharged patients from two hospitals. However, the experiences of patients and caregivers from other hospitals and in other countries are reported similar to what we found. ${ }^{19,24}$ Our sampled hospitals included a typical regional government hospital, similar to others in East Africa, and a private mission hospital. This mixture of health facilities provides as diverse a sample as could be derived from a single area. Further, as this qualitative survey was administered near the end of an ongoing study, differential recall, between caregivers whose children recently died compared to those whose children died earlier during the study, may have played a role in the kinds of events which were reported. However, this study used very general questions, and due to the importance of the events, it is unlikely that significant details were forgotten. Finally, this study did not directly ask about facilitators to health care seeking, which is an important aspect for overcoming barriers.

\section{Conclusion}

Out of hospital death is an important contributor to overall mortality in children discharged from hospital. Barriers contributing to death outside of hospitals are complex and include financial constraints for transportation, delayed care seeking, and a general unawareness of the risk surrounding the post-discharge period. These occur in the context of poor maternal education and environmental conditions. Interventions aimed at improving both the social context (eg. maternal education) and the health system (eg. emergency transport, post-discharge follow-up) may help to alleviate some of this burden.

\section{Acknowledgements}

The authors gratefully acknowledge the contributions of Annet Twinomuguni, Justine Kamazima, Agaba Collins, Clare Komugisha, Solome Kobugyenyi, Alexander Mutungi and Hassan Bariahikwa. Without whose dedication, this study could not have been completed.

\section{Conflict of interest}

None to declare.

\section{References}

1. Liu L, Oza S, Hogan D, et al. Global, regional, and national causes of child mortality in 2000-13, with projections to inform post-2015 priorities: an updated systematic analysis. Lancet. 2015;385(9966):430-440. doi:10.1016/ S0140-6736(14)61698-6.

2. Nair H, Simões E a F, Rudan I, et al. Global and regional burden of hospital admissions for severe acute lower respiratory infections in young children in 2010: a systematic analysis. Lancet. 2013;381(9875):1380-1390. doi:10.1016/S0140-6736(12)61901-1.

3. Veirum JE, Sodeman M, Biai S, Hedegård K, Aaby P. Increased mortality in the year following discharge from a paediatric ward in Bissau, Guinea-Bissau. Acta 
Paediatr. 2007;96(12):1832-1838. doi:10.1111/j.16512227.2007.00562.x.

4. Wiens M, Pawluk S, Kissoon N. Pediatric Post-Discharge Mortality in Resource Poor Countries: A Systematic Review. PLoS One. 2013;8(6):e66698. doi:10.1371/ journal.pone.0066698.

5. Bedford KJA, Sharkey AB. Local barriers and solutions to improve care-seeking for childhood pneumonia, diarrhoea and malaria in Kenya, Nigeria and Niger: A qualitative study. PLoS One. 2014;9(6):1-15. doi:10.1371/journal. pone.0100038.

6. Adedini SA, Odimegwu C, Bamiwuye O, Fadeyibi O, De Wet N. Barriers to accessing health care in Nigeria: Implications for child survival. Glob Health Action. 2014;7:1-10. doi:10.3402/gha.v7.23499.

7. Kadobera D, Sartorius B, Masanja H, Mathew A, Waiswa P. The effect of distance to formal health facility on childhood mortality in rural Tanzania, 2005-2007. Glob Health Action. 2012;5:1-9. doi:10.3402/gha.v5i0.19099.

8. Wiens MO. Childhood mortality from acute infectious diseases in Uganda: Studies in sepsis and post-discharge mortality. 2015.

9. MacQueen KM, McLellan E, Kay K, Milstein B. Codebook Development for Team-Based Qualitative Analysis. Cult Anthropol Methods. 1998;10(2):31-36. doi:10.1177/15 $25822 X 980100020301$.

10. Kayode GA, Adekanmbi VT, Uthman OA. Risk factors and a predictive model for under-five mortality in Nigeria: evidence from Nigeria demographic and health survey. BMC Pregnancy Childbirth. 2012;12(1):10. doi:10.1186/1471-2393-12-10.

11. Ayiko R, Antai D, Kulane A. Trends and determinants of under-five mortality in Uganda. East African Journal of Public Health. 2009; 6(2):136-40.

12. Ettarh RR, Kimani J. Determinants of under-five mortality in rural and urban Kenya. Rural Remote Health. 2012; 12:1812.

13. Rutherford ME, Mulholland K, Hill PC. How access to health care relates to under-five mortality in sub-Saharan Africa: Systematic review. Trop Med Int Heal. 2010;15(5):508-519. doi:10.1111/j.13653156.2010.02497.x.

14. Rutherford ME, Dockerty JD, Jasseh M, et al. Access to health care and mortality of children under 5 years of age in the Gambia: a case-control study. Bull World Health Organ. 2009;87(January):216-224. doi:10.2471/ BLT.08.052175.

15. Bazzano AN, Kirkwood BR, Tawiah-Agyemang C,
Owusu-Agyei S, Adongo PB. Beyond symptom recognition: Care-seeking for ill newborns in rural Ghana. Trop Med Int Heal. 2008;13(1):123-128. doi:10.1111/j.13653156.2007.01981.x.

16. Noordam AC, Carvajal-Velez L, Sharkey AB, Young M, Cals JWL. Care Seeking Behaviour for Children with Suspected Pneumonia in Countries in Sub-Saharan Africa with High Pneumonia Mortality. PLoS One. 2015;10:e0117919. doi:10.1371/journal.pone.0117919.

17. Taffa N, Chepngeno G. Determinants of health care seeking for childhood illnesses in Nairobi slums. Trop Med Int Heal. 2005;10(3):240-245. doi:10.1111/j.13653156.2004.01381.x.

18. Hill Z, Kendall C, Arthur P, Kirkwood B, Adjei E. Recognizing childhood illnesses and their traditional explanations: Exploring options for care-seeking interventions in the context of the IMCI strategy in rural Ghana. Trop Med Int Heal. 2003;8(7):668-676. doi:10.1046/j.13653156.2003.01058.x.

19. Sandberg J, Pettersson KO, Asp G, Kabakyenga J, Agardh A. Inadequate knowledge of neonatal danger signs among recently delivered women in southwestern rural Uganda: A community survey. PLoS One. 2014;9(5). doi:10.1371/journal.pone.0097253.

20. Wilson SE, Ouédraogo CT, Prince L, et al. Caregiver recognition of childhood diarrhea, care seeking behaviors and home treatment practices in rural Burkina Faso: a cross-sectional survey. PLoS One. 2012;7(3):e33273. doi:10.1371/journal.pone.0033273.

21. Rutebemberwa E, Kallander K, Tomson G, Peterson S, Pariyo G. Determinants of delay in care-seeking for febrile children in eastern Uganda. Trop Med Int Heal. 2009;14(4):472-479. doi:10.1111/j.13653156.2009.02237.x.

22. Houweling, T, Kunst, A. Socio-economic inequalities in childhood mortality in low- and middle-income countries: areview of the international evidence. British Medical Bulletin. 2010; 93(1):7-26. doi:10.1093/bmb/ldp048

23. Sonego M, Pellegrin MC, Becker G, Lazzerini M. Risk Factors for Mortality from Acute Lower Respiratory Infections (ALRI) in Children under Five Years of Age in Low and Middle-Income Countries: A Systematic Review and Meta-Analysis of Observational Studies. PLoS One. 2015;10:e0116380. doi:10.1371/journal.pone.0116380.

24. Geldsetzer P, Williams TC, Kirolos A, et al. The recognition of and care seeking behaviour for childhood illness in developing countries: A systematic review. PLoS One. 2014;9(4). doi:10.1371/journal.pone.0093427.

African Health Sciences Vol 16 Issue 4, December, 2016 